\title{
Prescription of Systemic Steroids for Acute Respiratory Infections in Korean Outpatient Settings: Overall Patterns and Effects of the Prescription Appropriateness Evaluation Policy
}

\author{
Taejae Kim', Young Kyung Do ${ }^{1,2}$ \\ ${ }^{1}$ Department of Health Policy and Management, Seoul National University College of Medicine, Seoul, Korea; ${ }^{2}$ Institute of Health Policy and \\ Management, Seoul National University Medical Research Center, Seoul, Korea
}

Objectives: The objective of this study was to identify individual and institutional factors associated with the prescription of systemic steroids in patients with acute respiratory infections and to investigate the role of a policy measure aimed to reduce inappropriate prescriptions.

Methods: We used data from the National Health Insurance Service-National Sample Cohort from 2006 to 2015 and focused on episodes of acute respiratory infection. Descriptive analysis and multiple logistic regression analysis were performed to identify individual-level and institution-level factors associated with the prescription of systemic steroids. In addition, steroid prescription rates were compared with antibiotic prescription rates to assess their serial trends in relation to Health Insurance Review and Assessment Service (HIRA) Prescription Appropriateness Evaluation policy.

Results: Among a total of 9460552 episodes of respiratory infection, the steroid prescription rate was 6.8\%. Defined daily doses/1000 persons/d of steroid increased gradually until 2009, but rose sharply since 2010. The steroid prescription rate was higher among ear, nose and throat specialties (13.0\%) than other specialties, and in hospitals (8.0\%) than in tertiary hospitals (3.0\%) and other types of institutions. Following a prolonged reduction in the steroid prescription rate, this rate increased since the HIRA Prescription Appropriateness Evaluation dropped steroids from its list of evaluation items in 2009. Such a trend reversal was not observed for the prescription rate of antibiotics, which continue to be on the HIRA Prescription Appropriateness Evaluation list.

Conclusions: Specialty and type of institution are important correlates of steroid prescriptions in cases of acute respiratory infection. Steroid prescriptions can also be influenced by policy measures, such as the HIRA Prescription Appropriateness Evaluation policy.

Key words: Inappropriate prescribing, Glucocorticoids, Respiratory tract Infections, Health Insurance Review and Assessment Service Prescription Appropriateness Evaluation

Received: April 28, 2019 Accepted: November 18, 2019

Corresponding author: Young Kyung Do, MD, PhD Department of Health Policy and Management, Seoul National University College of Medicine, 103 Daehak-ro, Jongno-gu,

Seoul 03080, Korea

E-mail: ykdo89@snu.ac.kr

This is an Open Access article distributed under the terms of the Creative Commons Attribution Non-Commercial License (https://creativecommons.org/licenses/by$\mathrm{nc} / 4.0 /$ ) which permits unrestricted non-commercial use, distribution, and reproduction in any medium, provided the original work is properly cited.

\section{INTRODUCTION}

The prescription practice of a medical provider is largely influenced by individual characteristics of the patient, such as sex, age, and income, as well as by institutional characteristics, such as institution type, specialty, and region [1-3]. Healthcare policy factors such as evaluation and monitoring of prescription appropriateness, feedback and public disclosure of the 
evaluation results, and graded payments based on evaluation results are also known to affect doctor's prescription practices [4]. The Health Insurance Review and Assessment Service (HIRA) Prescription Appropriateness Evaluation policy aims to improve prescription practices by comparing, analyzing, and providing feedback regarding current prescription practices [5]. The objective of this study was to identify individual and institutional factors associated with the prescription of systemic steroids in cases of acute respiratory infection (hereinafter referred to as a "cold"), as well as the role of the HIRA Prescription Appropriateness Evaluation policy in prescription decisions.

Like antibiotics, steroids are not effective in treating the symptoms of the common cold and may even cause side effects [4-6]. The established guidelines for the treatment of a cold recommend against prescribing either antibiotics or steroids [6,7]. Long-term use of steroids can undermine the immune system and/or cause Cushing syndrome, while studies have shown that even short-term use may increase the risk of developing septicemia, cardiac thrombosis, fracture, and other side effects [7]. Despite such risk factors, the prescription of steroids to common cold patients often occurs in clinical settings. According to 2007 data published by HIRA, the steroid prescription rate was $8.7 \%$ in 2006 [5]. Since then, the status of steroid prescriptions has been difficult to assess due to the lack of representative statistics and research on steroid prescriptions, and not much is known about the individual and institutional factors involved.

The HIRA Prescription Appropriateness Evaluation policy, which has been in effect since 2001, is relevant to the issue of the prescription of steroids to patients with colds. This program is acknowledged to have lowered the rate of antibiotic prescriptions for common cold patients [8-10], which fosters expectations that it has also lowered the rate of steroid prescriptions. Corticosteroids prescribed for respiratory diseases (Korean Standard Classification of Diseases [KCD-7], J00-J44, J47) were included in the policy's list of evaluation items starting in 2004, but were dropped from the list in 2009, constituting the only case in which a type of drug was removed from the evaluation item list. This provides an opportunity to understand how a policy has influenced prescription practices, as the trend of steroid prescriptions can be compared with that of antibiotics, which have been consistently included and were never removed from the evaluation list.

The goal of this study is to understand the individual and institutional factors that affect steroid prescriptions for common cold patients, as well as the effects of the HIRA Prescription Appropriateness Evaluation policy. More specifically, this study first analyzed the individual characteristics of patients (sex, age, initial or returning visit, and season) and institutional characteristics (institution type, specialty, and region), which are known to be factors influencing antibiotic prescriptions, to see whether they also influenced steroid prescriptions $[2,11]$. Second, this study used a time series analysis to compare the results with those observed for antibiotic prescription rates in order to understand the impact of the removal of steroids from the evaluation list in 2009.

\section{METHODS}

\section{Data and Study Population}

Data from the National Health Insurance Service-National Sample Cohort Database 2.0 (NHIS-NSC) were used for analysis. The NHIS-NSC data include information regarding birth, death, diagnosis and treatment, and health check-ups from 2002 to 2015 for a sample population selected from 48222537 Korean citizens in consideration of sex, age, region, and subscription to the National Health Insurance (NHI) program. The NHIS-NSC data integrated the diagnosis and treatment data of the population covered by Medicaid in 2006, but that same data is missing between 2002 and 2005. Given this situation, we chose to analyze the 2006-2015 data sets in this study.

Subjects of this study were identified by diagnosis codes that correspond to acute respiratory infection in the NHI claims data. The KCD-7 allocates codes J00-J06 to acute respiratory infection. Steroid prescription is not considered inappropriate for sinusitis (J01), laryngitis (J04), or epiglottitis (J05), according to existing studies and guidelines. The same is true for antibiotics [6,12-14]. Therefore, this study defined patients with codes $\mathrm{J} 00, \mathrm{~J} 02, \mathrm{J03}, \mathrm{J06}$, and $\mathrm{J} 39$ as common cold patients.

\section{Statistical Analysis}

The unit of analysis in this study is insurance claims for reimbursement for $\mathrm{NHI}$ services. A steroid prescription was defined as the prescription of drugs with code number 245 (corticosteroids) in accordance with the Korea Pharmaceutical Information Center drug classification table, and antibiotics were defined as the prescription of drugs with code number 610 (antibiotic agents). Prescribed routes of administration were limited to oral tablets and injections. The volume of prescribed steroids was calculated with reference to a previous study on an- 
tibiotic prescription volume [10]. Steroid product codes were matched with World Health Organization (WHO)-Anatomical Therapeutic Chemical codes based on the Anatomical Therapeutic Chemical table published by HIRA, and annual defined daily doses (DDD)/1000 people/d were calculated using the WHO's DDD index. Prescription rates of steroids and antibiotics were presented as the total annual number of visits divided by the number of visits with claims that include steroid and/or antibiotic prescriptions, based on the definition suggested in the HIRA Prescription Appropriateness Evaluation policy ([number of prescriptions of a drug/total number of visits] ${ }^{*} 100$ ) [15].

In order to analyze the individual and institutional characteristics at play, we first studied the individual (sex, age, insurance premium grade, and initial or returning visit) and institutional (season, specialty, type of institution, and region) characteristics that previous studies have suggested may affect antibiotic prescription $[2,11]$. After generating descriptive statistics for each characteristic, the chi-square test was performed to evaluate whether any statistically significant trends were observed among categories according to the investigated characteristics. Multiple logistic regression analysis was performed to identify factors affecting steroid prescriptions after account-

Table 1. General characteristics of the study population

\begin{tabular}{lc}
\hline Characteristics & $\mathbf{n}(\%)$ \\
\hline Total & $931002(100)$ \\
Sex & \\
Male & $445179(47.8)$ \\
Female & $485823(52.2)$ \\
Age (y) & \\
$0-10$ & $205796(22.1)$ \\
$10-19$ & $96526(10.4)$ \\
$20-29$ & $169248(18.2)$ \\
$30-39$ & $134522(14.4)$ \\
$40-49$ & $126431(13.6)$ \\
$50-59$ & $97259(10.4)$ \\
$60-69$ & $69109(7.4)$ \\
$\geq 70$ & $32111(3.4)$ \\
Income level & \\
1 (lowest) & $461988(49.6)$ \\
2 & $161190(17.3)$ \\
3 & $122580(13.2)$ \\
4 & $104579(11.2)$ \\
5 (highest) & $58740(6.3)$ \\
Missing & $21925(2.4)$ \\
\hline
\end{tabular}

Number refers to the number of individuals in the study population with any upper respiratory infection in the period examined. ing for the variables of initial/returning visit, season, specialty, type of institution, and region, as well as the patient's age, sex, and insurance premium grade.

A time series comparison of the rates of steroid and antibiotic prescriptions for certain conditions was carried out to understand the effects of removing steroids from the 2009 HIRA Prescription Appropriateness Evaluation list. The results were illustrated in a diagram. A difference-in-differences analysis to quantify the effects of the removal of steroids using the antibiotic prescription rate as a comparison group was not performed, as the purpose of this study was to understand general trends in prescription rates, which vary greatly across different types of medications. The descriptive analysis and regression analysis were conducted with SAS Enterprise Guide version 6.1 (SAS Institute Inc., Cary, NC, USA), and trends in the prescription rate were analyzed using Joinpoint Regression 4.7.0.0 (https://surveillance.cancer.gov/joinpoint/). A $p$-value less than 0.05 was considered to indicate statistical significance.

\section{Ethics Statement}

This study was approved by the Seoul National University Hospital Institutional Review Board (IRB No. E-1808-074-965).

\section{RESULTS}

The general characteristics of the study population are presented in Table 1. The number of total visits to a medical institution between 2006 and 2015 with symptoms of respiratory infection as the patient's main symptoms was 9460 552, after excluding missing and incorrect values from the original total of 10579258 visits. The entire population who visited a medi-

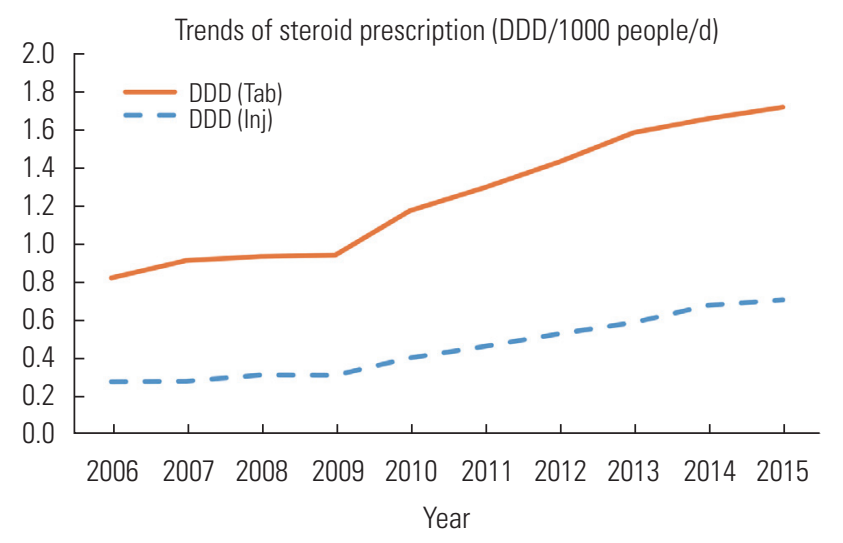

Figure 1. Trends in steroid prescription volume (DDD/1000 people/d). DDD, defined daily dose; Tab, tablet; Inj, injection. 
cal institution at least once for cold symptoms during that period was 931 002, with females (485 823 patients, 52.2\%), patients aged between 0 and 10 (205 796 patients, 22.1\%), and patients with insurance premium grade 1 (461 988 patients,
49.6\%) contributing the most to this group.

The trend in steroid prescriptions between 2006 and 2015, represented as the annual DDD/1000 people/d, is illustrated in Figure 1. In 2006, the steroid prescription volumes were 0.27

Table 2. General characteristics of upper respiratory infection cases and steroid prescriptions

\begin{tabular}{|c|c|c|c|}
\hline Characteristics & $\begin{array}{l}\text { Upper respiratory infection cases, } \\
\text { N (column \%) }\end{array}$ & $\begin{array}{l}\text { Steroid prescriptions, } \\
\text { n }(\%, n / N)\end{array}$ & $\begin{array}{l}\text { Logistic regression of steroid prescription, } \\
\text { OR (95\% confidence interval) }\end{array}$ \\
\hline Total & $9460552(100)$ & 645348 (6.8) & \\
\hline \multicolumn{4}{|l|}{ Type of visit } \\
\hline First visit & 4634800 (49.0) & 309141 (6.7) & 1.00 (reference) \\
\hline Return visit & 4813672 (50.9) & $335256(7.0)$ & $1.23(1.23,1.23)$ \\
\hline \multicolumn{4}{|l|}{ Season } \\
\hline Summer & 1728707 (18.3) & $114729(6.6)$ & $1.06(1.05,1.06)$ \\
\hline Autumn & 2587698 (27.4) & $183689(7.1)$ & $1.09(1.09,1.09)$ \\
\hline Winter & 2559616 (27.1) & 171691 (6.7) & $0.96(0.96,0.96)$ \\
\hline \multicolumn{4}{|l|}{ Specialty } \\
\hline General practice & 1999656 (21.1) & 156877 (7.8) & 1.00 (reference) \\
\hline Family medicine & 392255 (4.1) & 24245 (6.2) & $0.82(0.82,0.82)$ \\
\hline Other & 450184 (4.8) & $29923(6.6)$ & $0.72(0.72,0.73)$ \\
\hline \multicolumn{4}{|l|}{ Type of institution } \\
\hline Tertiary hospital & $21211(0.2)$ & $627(3.0)$ & 1.00 (reference) \\
\hline General hospital & $191713(2.0)$ & $11946(6.2)$ & $2.77(2.70,2.83)$ \\
\hline Hospital & 439964 (4.7) & 35031 (8.0) & $4.83(4.72,4.91)$ \\
\hline Clinic & 8772474 (92.7) & 594853 (6.8) & $2.28(2.33,2.44)$ \\
\hline Other & $35190(0.4)$ & 2891 (8.2) & $3.82(3.72,3.92)$ \\
\hline \multicolumn{4}{|l|}{ Residential area } \\
\hline Seoul & 1899498 (20.1) & 143707 (7.6) & 1.00 (reference) \\
\hline Gyonggi & 2265018 (23.9) & 166095 (7.3) & $1.06(1.06,1.07)$ \\
\hline Gangwon & 262835 (2.8) & 15864 (6.0) & $0.87(0.87,0.87)$ \\
\hline Chungbuk, Chungnam & 698444 (7.4) & 46422 (6.6) & $1.00(0.99,1.00)$ \\
\hline Junbuk, Junnam & 688499 (7.3) & $39343(5.7)$ & $0.77(0.72,0.73)$ \\
\hline Gyeongbuk, Gyongnam & 1016138 (10.7) & 69025 (6.8) & $0.98(0.98,0.99)$ \\
\hline Jeju & 118399 (1.3) & $8450(7.1)$ & $1.06(1.05,1.07)$ \\
\hline Sex & & & Included \\
\hline Age & & & Included \\
\hline Income & & & Included \\
\hline
\end{tabular}

$\mathrm{N}$ refers to the number of upper respiratory infection cases in the period examined, while $\mathrm{n}$ refers to the number of upper respiratory infection cases with a steroid prescription in the period examined.

Individual factors (sex, age, and income quintile) were included in the multiple logistic regression analysis but are not presented here. 


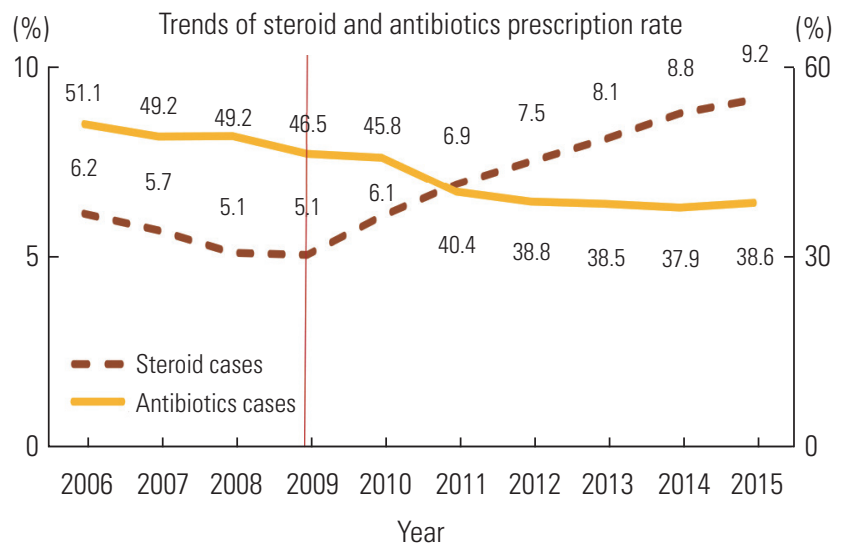

Figure 2. Trends in steroid and antibiotic prescription rates from 2006 to 2015. The left axis represents the steroid prescription rate, and the right axis the antibiotic prescription rate. The vertical red line denotes the year when steroids ceased to be listed as part of the Health Insurance Review and Assessment Service Prescription Appropriateness Evaluation policy.

and $0.82 \mathrm{DDD} / 1000$ people/d for injections and tablets, respectively; from there, a slight upward trend continued until 2009, when these volumes reached 0.30 and 0.93 DDD/1000 people/d. After 2009, the upward trend accelerated to reach 0.70 and $1.72 \mathrm{DDD} / 1000$ people/d in 2015.

The rates of steroid prescriptions by institutional factors are provided in Table 2. The overall steroid prescription rate was $6.8 \%$, but this rate varied significantly by specialty and institution type. Ear, nose and throat (ENT) specialists had the highest steroid prescription rate, at $13.0 \%$, and pediatricians had the lowest, at $3.4 \%$. Hospitals prescribed steroids at the highest rate (8.0\%), while tertiary hospitals prescribed them at the lowest rate (3.0\%). Type of visit, season, and region of the institution were not correlated as strongly with the steroid prescription rate as were specialty and institution type. Those two factors maintained statistical significance in the results of the multiple logistic regression analysis, which accounted for visit type, season, region, patient age, sex, and income level.

Figure 2 shows the trends for the annual prescription rates of steroids and antibiotics from 2006 to 2015. Over that period, the antibiotic prescription rate dropped consistently, from 51.1\% in 2006 to $38.6 \%$ in 2015. In comparison, the steroid prescription rate dropped from 2006 to 2009 at an average rate of 0.4 percentage points per year, reaching a value of $5.1 \%$ in 2009 ; however, this rate then increased at an average of 0.7 percentage points per year starting in 2010, the year after the removal of the drugs from the HIRA Prescription Appropriateness Eval-

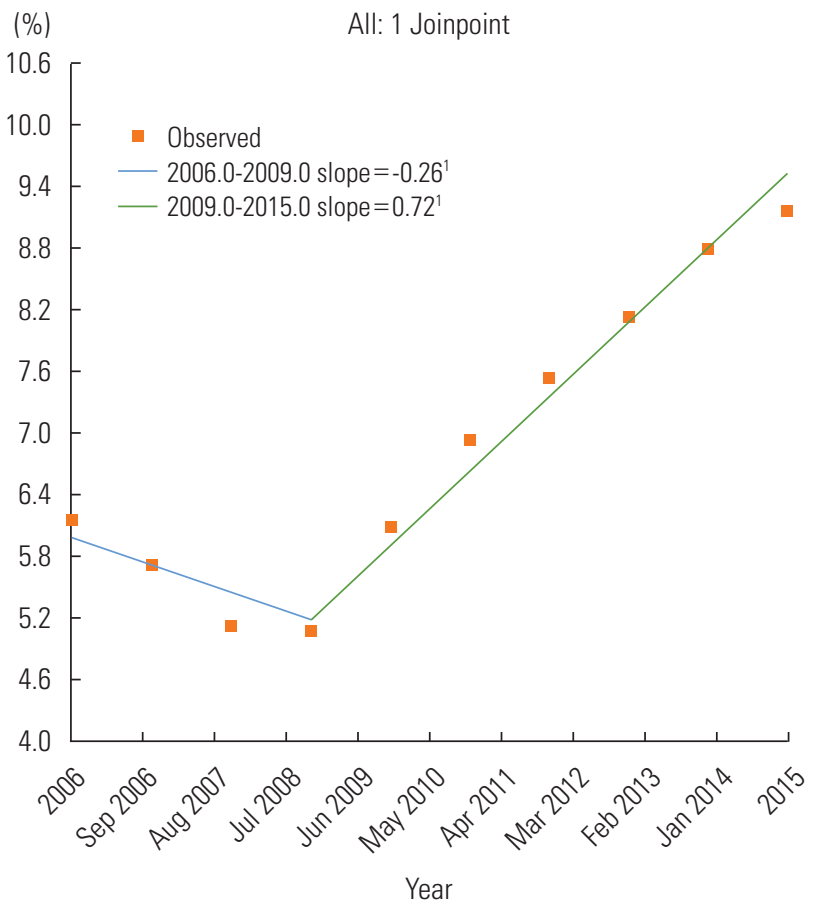

Figure 3. Joinpoint regression graph of steroid prescription rates from 2006 to 2015. Final selected model: 1 Joinpoint. 'Indicates that the slope is significantly different from zero at the alpha $=0.05$ level.

uation policy, and reached $9.2 \%$ in 2015 . The Joinpoint regression graph of the steroid prescription rate also indicated that 2009 was the statistically significant $(p<0.001)$ inflection point. The slope was -0.26 before 2009 , indicating a decreasing trend, but then switched to an increasing trend with a slope of 0.72 (Figure 3).

\section{DISCUSSION}

This descriptive study was conducted to investigate the status of steroid prescriptions for cold patients, to identify any correlations between the prescription rate and individual or institutional characteristics, and to assess the effects of the HIRA Prescription Appropriateness Evaluation policy. The results can largely be summarized in two statements. First, when examining the prescription rates based on patient-level and institutional characteristics, the same factors that were found in previous studies to encourage more prescriptions of antibiotics, such as specialty (ENT, 13\%) and institution type (hospitals, 8.0\%), were observed to have the same effects on steroid prescriptions [11]. Second, the steroid prescription rate dropped from $6.2 \%$ in 2006 to $5.1 \%$ in 2009 , but rose after the drugs were excluded 
from the evaluation list, reaching 9.2\% in 2015.

The marked differences in steroid prescription rates by institution type indicate that expert groups, relevant organizations, and policy-makers should redouble their efforts. Among specialties, ENT had the highest rate of steroid prescription (13.0\%) and accounted for a large proportion of visits (22.0\%). Previous studies of antibiotic prescriptions in cold patients $[2,11]$ also suggested a higher rate of prescriptions by ENT specialists. An analysis of steroid prescription rates by type of institution showed that tertiary hospitals prescribed the drugs at the lowest rate (3.0\%) - notably, this rate was lower than that of less specialized institutions. This implies that hospitals (8.0\%) and clinics $(6.8 \%)$ can improve by setting the prescription rate attained in tertiary hospitals as an achievable benchmark.

In relation to the HIRA Prescription Appropriateness Evaluation policy, the rate and volume of steroid prescriptions for cold patients decreased until 2009, when the drugs were still subject to evaluations, but began to increase in 2010, after removal from the list. In comparison, antibiotics continued to be evaluated under the policy, and previous studies have confirmed a consistent decline in prescription rates $[8,9]$. The Joinpoint regression analysis results reaffirmed that 2009 was the significant inflection point for steroid prescription rate (Figure 3). Although it is known that performance reporting, such as stroke appropriateness evaluation, may influence evaluation indices and the behavior of medical providers [16], the specific mechanism underlying this influence is not clear. A study from the United States suggested that disclosure of evaluation scores may eventually transform clinical practices by influencing patients' choice of institution and by incentivizing institutions to work harder to get better scores [17], but such a mechanism does not apply to steroid prescriptions for cold patients, since the evaluation results are not disclosed. The evaluation or the performance reporting may have encouraged self-regulation among providers [18], but this is also difficult to verify. The adjustment of reimbursement payouts announced along with the evaluation may have had an effect, but this hypothesis is not supported [19].

We acknowledge that this study has limitations, as follows. First, "cold" was defined as cases listing the KCD-7 code as the main diagnosis in reimbursement claims, but a patient's actual disease may not have matched the main diagnosis in their claim. Second, patients with underlying conditions requiring steroid prescriptions who presented with a cold as their main concern might have been appropriately prescribed steroids to treat their underlying conditions, but this possibility could not be excluded. Third, a provider's steroid prescription practices may depend on the type of institution where they were trained, but this could not be validated statistically with the currently available data. Lastly, the observed effects of removing steroids from the HIRA evaluation list would have been more compelling if all other possible factors had been controlled for, but this study instead performed a time series analysis to compare between steroids and antibiotics. Although the timeline and rebounding trends clearly align with this hypothesis, in the absence of any other obvious explanations, this hypothesis should be evaluated in well-designed future studies.

Despite the above limitations, this study is noteworthy in that it presented the current status of steroid prescription rates for cold patients using representative sample cohort data. To summarize the results of this study, steroid prescription rates differed significantly by specialty and institution type, even after accounting for individual and institutional characteristics. This suggests the necessity and possibility of enhancing the quality of medical service with regards to prescriptions. Moreover, the steroid prescription rate began to increase over time immediately after steroids were excluded from the HIRA Prescription Appropriateness Evaluation list, which underscores how policy factors can play an important role in prescription practices.

\section{CONFLICT OF INTEREST}

The authors have no conflicts of interest associated with the material presented in this paper.

\section{FUNDING}

None.

\section{ACKNOWLEDGEMENTS}

None.

\section{AUTHOR CONTRIBUTIONS}

Conceptualization: TK, YKD. Data curation: TK, YKD. Formal analysis: TK. Funding acquisition: None. Writing - original draft: TK, YKD. Writing - review \& editing: YKD, TK. 


\section{ORCID}

Taejae Kim https://orcid.org/0000-0001-9409-9352

Young Kyung Do https://orcid.org/0000-0001-5024-8264

\section{REFERENCES}

1. Lee YS, Kim MK, Kim YI, Shin YS, Lee HJ, Ahn HS. Private practitioners' antimicrobial prescription patterns for acute respiratory infections in children. J Korean Public Health Assoc 1991; 17(2):3-19 (Korean).

2. Kim NS, Jang SN, Jang SM. Factors influencing antibiotics prescribing of primary health physicians in acute upper respiratory infections. J Prev Med Public Health 2005;38(1):1-8 (Korean).

3. Jo C, Lim JY, Lee SY. The effect of the degree of competition of the hospital market regions on clinic's rate of antibiotics prescription. KDI J Econ Policy 2008;30(2):129-155 (Korean).

4. Kim YI, Shin YS. Health policy and management. Seoul: Seoul National University Press; 2017, p. 397-398 (Korean).

5. Health Insurance Review and Assessment Service. Evaluation project on appropriate prescribing report 2006. Seoul: Health Insurance Review and Assessment Service; 2007, p. 3 (Korean).

6. Kim SW. Upper respiratory infections in adults. J Korean Med Assoc 2010;53(1):10-19 (Korean).

7. Kim SY. Drug therapy for the common cold. J Korean Med Assoc 2015;58(2):147-153 (Korean).

8. Kim SK, Kim HE, Back MS, Lee SH. The effect of public report on antibiotics prescribing rate. Korean J Clin Pharm 2010;20(3): 242-247 (Korean).

9. Jung $K$, Jun $D$, Kim H. The impact of the disclosure of public information on prescription antibiotics at individual hospitals in Seoul. Korean J Public Adm 2008:46(1):123-150 (Korean).
10. Chae SM, Park EJ, Park S. Antibiotic consumption and expenditures for acute upper respiratory tract infections in outpatients. Yakhak Hoeji 2013;57(3):199-204 (Korean).

11. Choi WJ, Yim E, Kim TH, Suh HS, Choi KC, Chung W. Analysis of factors related to the prescription of antibiotics for the acute upper respiratory infection. Health Policy Manag 2015;25(4): 256-263 (Korean).

12. Ahn JH, Woo WK, Kim YS, Song YJ, Jeon IK, Jung JR, et al. Efficacy of adjuvant short term oral steroid therapy for acute pharyngitis. Korean J Otorhinolaryngol-Head Neck Surg 2003; 46(11):971-974 (Korean).

13. Yoon YK, Park CS, Kim JW, Hwang K, Lee SY, Kim TH, et al. Guidelines for the antibiotic use in adults with acute upper respiratory tract infections. Infect Chemother 2017;49(4):326-352.

14. Arroll B, Kenealy T. Antibiotics for the common cold and acute purulent rhinitis. Cochrane Database Syst Rev 2005;(3):CD000247.

15. Health Insurance Review and Assessment Service. Evaluation project on appropriate prescribing report. Seoul: Health Insurance Review and Assessment Service; 2009, p. 6 (Korean).

16. Choi EY, Ock M, Lee HJ, Jo MW, Lee SI. Analyzing the quality assessment program for acute stroke of the Health Insurance Review and Assessment Service. J Health Tech Assess 2017; 5(1):42-48 (Korean).

17. Hibbard JH, Stockard J, Tusler M. Hospital performance reports: impact on quality, market share, and reputation. Health Aff (Millwood) 2005;24(4):1150-1160.

18. Healy J, Braithwaite J. Designing safer health care through responsive regulation. Med J Aust 2006;184(S10):S56-S59.

19. Yoon HJ , Park EC. Reviews of pay-for-performance and suggestion for Korean value incentive program. Health Policy Manag 2017;27(2):121-127 (Korean). 